\title{
Diet, anthropometric measures and prostate cancer risk: a review of prospective cohort and intervention studies
}

Citation for published version (APA):

Dagnelie, P. C., Schuurman, A. G., Goldbohm, R. A., \& van den Brandt, P. A. (2004). Diet, anthropometric measures and prostate cancer risk: a review of prospective cohort and intervention studies. BJU International, 93(8), 1139-50. https://doi.org/10.1111/j.1464-410X.2004.04795.x

Document status and date:

Published: 01/01/2004

DOI:

10.1111/j.1464-410X.2004.04795.x

Document Version:

Publisher's PDF, also known as Version of record

Please check the document version of this publication:

- A submitted manuscript is the version of the article upon submission and before peer-review. There can be important differences between the submitted version and the official published version of record.

People interested in the research are advised to contact the author for the final version of the publication, or visit the DOI to the publisher's website.

- The final author version and the galley proof are versions of the publication after peer review.

- The final published version features the final layout of the paper including the volume, issue and page numbers.

Link to publication

\footnotetext{
General rights rights.

- You may freely distribute the URL identifying the publication in the public portal. please follow below link for the End User Agreement:

www.umlib.nl/taverne-license

Take down policy

If you believe that this document breaches copyright please contact us at:

repository@maastrichtuniversity.nl

providing details and we will investigate your claim.
}

Copyright and moral rights for the publications made accessible in the public portal are retained by the authors and/or other copyright owners and it is a condition of accessing publications that users recognise and abide by the legal requirements associated with these

- Users may download and print one copy of any publication from the public portal for the purpose of private study or research.

- You may not further distribute the material or use it for any profit-making activity or commercial gain

If the publication is distributed under the terms of Article 25fa of the Dutch Copyright Act, indicated by the "Taverne" license above, 


\title{
Diet, anthropometric measures and prostate cancer risk: a review of prospective cohort and intervention studies
}

\author{
P.C. DAGNELIE, A.G. SCHUURMAN, R.A. GOLDBOHM* and P.A. VAN DEN BRANDT \\ Department of Epidemiology, Maastricht University, and *TNO Nutrition and Food Research Institute, Zeist, the Netherlands
}

Accepted for publication 28 January 2004

\section{ABSTRACT}

We reviewed 37 prospective cohort and four intervention studies on potential dietary risk factors for prostate cancer, published between 1966 and September 2003. Some studies were limited by small size, crude measurement of dietary exposure and limited control for confounders. Intervention and prospective cohort studies support a protective role against prostate cancer for selenium, and possibly for vitamin $E$, pulses and tomatoes/lycopene. Overall consumption of meat, eggs, vegetables, fruit, coffee, tea, carotenoids and vitamins $A, C$ and $D$ was not consistently related to prostate cancer risk. Intervention studies also indicate that supplementation with $\beta$-carotene does not lower prostate cancer risk, except possibly in men with low $\beta$-carotene status at baseline. For specific types of meat, alcoholic drinks, dairy products, fat and anthropometric measures, most cohort studies suggest either an increased risk or no relation with prostate cancer. For calcium, two cohort studies suggest an increased risk at very high calcium intakes (>2000 mg/day). In conclusion, prospective studies are consistent with a protective role for selenium, and possibly vitamin $E_{1}$ pulses and tomatoes/lycopene, in the aetiology of prostate cancer. Studies are inconclusive on the role of meat, dairy products, fat, vegetables, fruits, alcohol and anthropometric measures, whereas a very high calcium intake appears to be positively associated with prostate cancer risk.

\section{INTRODUCTION}

Prostatic cancer is presently one of the most frequently occurring forms of cancer in Western countries. In Europe, and in the USA and Canada, the age-adjusted incidence is increasing [1]. Prostate cancer occurrence differs markedly across different populations; age-adjusted incidence rates of clinical prostate cancer of $\approx 1$ per 100000 are reported for China, whereas in the USA rates for whites are 45-65 per 100 000, and rates for blacks are as high as 102 per 100000 . Incidence rates in Western Europe are 20-30 per 100 000, with some variation among countries [2]. Mortality rates have not changed markedly during the past century [1]. In contrast to clinical prostate cancer incidence, which varies geographically, latent prostate carcinomas seem to be equally distributed across areas with high and low total prostate cancer incidence rates [3-5], supporting the view that environmental factors may be important in the progression from latent to clinical prostate carcinoma.

Dietary factors are among the environmental variables which may be important in the causes of prostate cancer. Since 1980 many reviews on the relation between dietary factors and prostate cancer have been published, e.g. [6-11], but the scope of most reviews was limited in the number of dietary risk factors, the broadness of dietary categories, and/or the completeness of included studies.

The present review discusses both methodological issues and results on individual foods/nutrients; it is limited to prospective cohort and intervention studies, as such studies are less prone to bias than, e.g. case-control and ecological studies.

\section{METHODS}

Published papers were searched using Medline/Pubmed (the computerized database from the Index Medicus) using the keywords 'epidemiology', 'etiology', 'diet', 'risk factor', 'cohort', and 'study', all combined with 'prostatic neoplasms.' Furthermore, references in the selected publications were checked for relevant publications not included in the Medline/Pubmed search. Studies included in this review are prospective cohort and intervention studies published between 1966, the earliest year covered by Medline, and September 2003. Only studies in which dietary intake was measured by blood biomarkers, questionnaires or interviews are included. Dietary factors described include vegetables, fruit, meat, fish, milk and dairy products, eggs, coffee, tea, vitamins, fat, fatty acids, selenium and alcohol. In addition, prospective studies on anthropometric measures as a risk factor for prostate cancer are reviewed. In most cases, reported risk ratios are adjusted for age but not for other risk factors, as this information was not available in most publications. Items are considered to be positively or negatively associated with prostate cancer risk when the relative risk (RR) estimate for the highest vs lowest exposure category was $<0.8$ (decrease) or $>1.2$ (increase). $P<0.05$ was considered to indicate statistical significance.

\section{RESULTS}

Four randomized, placebo-controlled intervention studies [12-17] were identified, three in the USA $[12-15,17]$ and one in Finland [16] (Table 1). The following supplements were studied in these trials: $\beta$-carotene [12-14]; $\beta$-carotene with/without vitamin $E_{1}$ in a factorial design [16]; $\beta$ carotene with retinol [17], and selenium [15]. In three of these four trials prostatic cancer was a secondary outcome measure; two trials were in subjects at high risk of developing lung cancer $[16,17]$ and one in subjects at high risk of basal/squamous cell carcinoma of the skin [15].

In all, 37 prospective cohort studies (including reports of prospective cohort analyses within intervention studies) were identified; 17 of 
TABLE 1 Descriptive characteristics of published intervention studies on dietary risk factors for prostate cancer, according to year of baseline measurement; all studies used a placebo comparison.

\begin{tabular}{|c|c|c|c|c|c|c|c|}
\hline Country Ref & Baseline & $\begin{array}{l}\text { Cohort size } \\
\text { (age range)* }^{*}\end{array}$ & $\begin{array}{l}\text { Follow-up, } \\
\text { years }\end{array}$ & $\begin{array}{l}\text { No. of cases } \\
\text { (I or D)† }\end{array}$ & Design & Intervention frequency & Notes \\
\hline USA [12-14] & 1982 & $22071(40-84)$ & 12.9 & $\begin{array}{c}1047-1117 \\
(1+D)\end{array}$ & $2 \times 2 \mathrm{~F}$ & $\begin{array}{r}\beta \text {-carotene/P } 50 \mathrm{mg} \mathrm{AD} \\
\text { aspirin/P } 325 \mathrm{mg} \mathrm{AD \neq}\end{array}$ & Physicians \\
\hline USA [15] & 1983 & $974(63) \uparrow$ & 6.5 & $48(I)$ & $B R / t+$ clinic & Selenium 200 mg D & $\begin{array}{l}\text { Patients with previous } \\
\text { skin cancer }\end{array}$ \\
\hline Finland [16] & $1985-88$ & 29133 (50-69) & $5-8$ & 246 (I) & $2 \times 2 F$ & $\begin{array}{l}\alpha \text {-tocopherol } 50 \mathrm{mg} \mathrm{D} \\
\beta \text {-carotene } 20 \mathrm{mg} \mathrm{D}\end{array}$ & Smokers \\
\hline USA [17] & 1988 & 18314 (58) & 4 & $300(I)$ & $\begin{array}{l}\text { Part } 2 \times 2 \mathrm{~F} \\
\quad \text { Part } 1: 1\end{array}$ & $\begin{array}{l}\beta \text {-carotene } 30 \mathrm{mg} \mathrm{D} \\
25000 \mathrm{IU} \\
\text { vitamin A D }\end{array}$ & $\begin{array}{l}\text { Men at high risk of } \\
\text { lung cancer }\end{array}$ \\
\hline
\end{tabular}

F, factorial design; $A D$, alternate days; $D$, daily; $B R$, block randomization; $P$, placebo; *Age range of the study population; +1 , incident cases, $D$ deceased cases; FAspirin/placebo: $325 \mathrm{mg}$ on alternate days from 1982 to 1988 when the aspirin/placebo intervention was terminated early because of a $44 \%$ reduction in incidence of first myocardial infarction by aspirin; the $\beta$-carotene intervention was continued until 1995; $\uparrow$ Mean age.

these studies had used biomarkers in blood or toenails [18-52] as indicators of dietary intake, with or with no dietary questionnaires (Table 2), whereas 20 studies had used dietary questionnaires or interviews only [41,53-80] (Table 3). Baseline measurements in most of the cohort studies had been taken before 1980. The cohort size varied from 974 [15] to 450279 men [71]. Reports of eight studies included $>400$ cases $[12-14,37,42-46,49$ $54,72-80]$, whereas eight included $<50$ cases $[15,18-21,23,25,28,36]$ and another seven $50-100$ cases $[26,27,55,58,63,67,70]$. The largest number of cases to date is 3811 [80]. In 24 studies incidence was used as an endpoint, in eight mortality and in nine both incidence and mortality. Most reports did not mention whether the concerned dietary questionnaire had been validated; only reports on the Health Professionals Follow-Up Study [41,72-79], the Netherlands Cohort Study on Diet and Cancer [42-45,49,51], the cohort-based part of the Alpha-Tocopherol Beta-Carotene Cancer Prevention Study (ATBC Study) $[40,47]$, the Cancer Prevention Study II Nutrition Cohort [80], and a Norwegian study [67] mentioned that the questionnaire used had been validated. These five studies had used relatively extensive dietary questionnaires (68-276 items).

In Tables 4-6 results from intervention and cohort studies on dietary risk factors for prostate cancer are summarized. A RR of 1 indicates null association, $>1$ a positive association (i.e. increased risk), and $<1$ a negative association (i.e. reduced risk/ protective effect). Table 4 gives results from four published trials on $\beta$-carotene, retinol, vitamin E and selenium. In the ATBC Study [16] and the Physicians' Health Study (PHS) [12-14], there was no effect of supplementation with $\beta$-carotene. The ATBC study was performed in smokers and the PHS both smokers and nonsmokers. Within the PHS the effect of $\beta$-carotene supplementation on prostate cancer incidence was similar in smokers and nonsmokers (both $R R=1.0$ ) [12]. However, $\beta$ carotene supplementation seemed to reduce prostate cancer incidence in subjects with low baseline plasma $\beta$-carotene levels $(R R=0.68$, Table 4), but to increase prostate cancer incidence in subjects with high baseline levels $(R R=1.33)$ [13]. The Beta-Carotene and Retinol Efficacy Trial showed no effect of supplementation with $\beta$-carotene and retinol in smokers [17].

Significantly reduced risks were reported for on supplementation with vitamin $\mathrm{E}$ in the ATBC Study in smokers [16], and for selenium in the Nutritional Prevention of Cancer Trial in subjects at high risk of developing basal/ squamous cell carcinoma of the skin [15].

Results from prospective cohort studies with blood or toenail samples as biomarkers are also shown in Table 4, and from studies with questionnaires in Table 5. For vitamin A/ retinol the results were inconsistent; three cohort studies with blood samples showed a decreased risk of prostate cancer [26-28], one showed no association [20] and five showed an increased risk $[19,21,25,36,46]$. In questionnaire-based studies of vitamin A/retinol intake, five null associations $[51,60,69,71,78]$, one negative [51] and one positive [78] association were reported.

For carotenoids, serum-based studies showed a reduced prostate cancer risk for $\alpha$-carotene [46]; no effect [26] or an increased risk [25] for $\beta$-carotene, and no association for $\beta$ cryptoxanthin [46]. In questionnaire-based studies, there was no association for intake of $\alpha$-carotene $[51,78] \beta$-carotene $[51,56,60,69,78]$, or lutein/zeaxanthin $[51,78]$; for $\beta$-cryptoxanthin, one study showed no effect [78] and one a significantly increased risk [51]. Lycopene showed an inverse association with prostate cancer risk in two serum-based $[26,46]$ and one questionnairebased [78] study, but no association in two other questionnaire-based studies $[51,73]$.

Other vitamins investigated in cohort studies include C, D and E. Vitamin C showed no association with prostate cancer risk in one serum-based study [36]; three questionnairebased studies showed no association for vitamin C intake from supplements $[51,69]$ or food $[69,78]$, but two questionnaire-based studies showed an increased risk for vitamin C intake $[51,56]$ and one a reduced risk for vitamin C from supplements [51]. For the vitamin D-metabolite 25-OH-D measured in blood samples, no association with prostate cancer risk was apparent [29,34], whereas 1,25-OH-D showed either an inverse association [29] or no association [34] with 
TABLE 2 Descriptive characteristics of published prospective cohort studies on prostate cancer and dietary and anthropometric risk factors measured by biomarkers (blood/toenails) with or without questionnaires/interviews, according to year of baseline measurement

\begin{tabular}{|c|c|c|c|c|c|c|c|c|}
\hline $\begin{array}{l}\text { Country } \\
\text { Ref }\end{array}$ & Baseline & $\begin{array}{l}\text { Cohort size } \\
\text { (age range)* }\end{array}$ & $\begin{array}{l}\text { Follow-up, } \\
\text { years }\end{array}$ & $\begin{array}{l}\text { No. of cases } \\
(\text { I or D) }+\end{array}$ & $\begin{array}{l}\text { Exposure } \\
\text { measure }\end{array}$ & $\begin{array}{l}\text { Investigated§ } \\
\text { exposure variables }\end{array}$ & $\begin{array}{l}\mathrm{N} \text { items on } \\
\text { diet (alcohol) }\end{array}$ & $\begin{array}{l}\text { Validation } \\
\text { dietary } 0\end{array}$ \\
\hline USA [18] & $1960-62$ & $3102(>40)$ & $12-14$ & $8(I+D)$ & BS & V & - & - \\
\hline USA $[29,33]$ & 1964-71 & $250000 \neq(38-81)$ & $1-23$ & $181(\mathrm{I})$ & BS & V & - & - \\
\hline USA $[22,24,31,38]$ & 1965-68 & 7999 (>65) & $21-23$ & $174-306$ (I) & $\begin{array}{c}\text { BS; FFO (interview) } \\
\text { 24-h recall }\end{array}$ & $V, 1-6,14, A, A P$ & 23 & unknown \\
\hline USA [20] & 1967-69 & $?$ & $10-12$ & $14(I+D)$ & BS & V & - & - \\
\hline Finland $[23,25]$ & $1968-72$ & $36265 \neq(15-99)$ & 8 & $32(I)$ & $\mathrm{BS} ; \mathrm{O}$ & V & - & - \\
\hline Switzerland [36] & $1971-73$ & 2974 (?) & 17 & 30 (D) & $\mathrm{BS} ; 0$ & V & - & - \\
\hline USA [27] & $1971-75$ & $2440(>50)$ & 10 & $84(I+D)$ & $\mathrm{BS} ; \mathrm{O}$; exam & V & - & - \\
\hline USA [21] & $1972-76$ & 6167 (?) & $0-10$ & $13(I)$ & $\mathrm{BS}$; interview & $V_{1} 11$ & - & - \\
\hline USA [28] & $1972-76$ & 8825 (>30) & 8.5 & 6 (D) & $\mathrm{BS}$; interview & $V_{1} 11$ & - & - \\
\hline Sweden [30] & 1972-78 & $53242(20-55)$ & 16.3 & $220(I)$ & BS; O; exam & $8, A P$ & - & - \\
\hline USA [19] & 1973-74 & 4480 (30-69) & 5 & $11(\mathrm{I})$ & BS & V & - & - \\
\hline Norway [39] & 1973-94 & $?(50)$ & $0.4-19.2$ & $141(\mathrm{I})$ & BS & 7,8 & - & - \\
\hline USA $[26,35,85]$ & 1974 & 25802 (?) & $12 ?$ & 43-103 (I) & BS & $8, \mathrm{~V}$ & - & - \\
\hline USA $[32,34,37,46,50]$ & 1982 & $22071(40-84)$ & $6-11$ & 120-1 047ף (I) & $\mathrm{BS} ; 0$ & $3,5,8, V, 12, A P$ & 131 & unknown \\
\hline Finland $[40,47]$ & 1985-93 & 29133 (50-69) & 7 & 233-317 (I) & $\mathrm{BS} ; \mathrm{FFO}$ & $V, 11,12,15$ & 276 & yes \\
\hline USA [41,72-79] & 1986 & 51529 (40-75) & $6-12$ & 300-2482 (I + D) & TC; FFO & $1-8, V, A P$ & 131 & yes \\
\hline $\begin{array}{l}\text { Netherlands } \\
\quad[42-45,49,51,52]\end{array}$ & 1986 & 58279 (55-69) & 6.3 & $704(I)$ & TC; FFO & $1-12, V, A, A P$ & 150 & yes \\
\hline USA [48] & 1986 & 10456 & $0-7$ & $145(I)$ & $\mathrm{BS} ; \mathrm{TC} ; \mathrm{FFO}$ & $V_{1} 11$ & - & - \\
\hline
\end{tabular}

$B S$, blood sample; $A$, alcohol, $V$, vitamins; $Q$, questionnaire; $A P$, anthropometry; FFQ, food frequency questionnaire; TC, toenail clipping; *Age range of the study population; +1 , incident cases, $D$ deceased cases; $\neq$ Only a total of men and women given; $\$$ Number of cases depending on exposure under study. $\$ 1$ vegetables, 2 fruits, 3 meat, 4 fish, 5 dairy, 6 eggs, 7 fat, 8 fatty acids, 9, cheese, 10 protein, 11 selenium, 12 calcium, 14 coffee, 15 phosphorus.

prostate cancer risk. In a single questionnairebased study, vitamin D intake showed no association [80].

Serum levels of vitamin $E$ and $\alpha$-tocopherol showed a negative association with prostate cancer risk in three studies $[19,23,48]$, and no relation in one [26]. Vitamin $E$ intake from food $[40,51,78]$ and supplements $[40,69,75]$ showed no association with prostate cancer risk, except for one study which showed a reduced risk for vitamin $E$ from supplements [51]. High $\gamma$-tocopherol levels in serum were associated with a significantly reduced prostate cancer risk $(R R=0.19)$ in one study [48].

Selenium was associated with a decreased prostate cancer risk in five of six biomarkerbased studies, i.e. two serum-based studies $[21,28]$ and three based on selenium analysis of toenails $[41,48,52]$; only one serum-based study [40] showed no effect.

Calcium intake showed no association with prostate cancer risk in two questionnaire- based studies $[45,47]$; in these, mean calcium intake in the highest quintile was 1330 and $1840 \mathrm{mg} /$ day, respectively. In contrast, in two other questionnaire-based studies $[76,80]$, a calcium intake of $>2000 \mathrm{mg} /$ day was associated with a marked increase in prostate cancer risk; in both studies, calcium from food sources and from supplements were independently associated with increased prostate cancer risk.

Intake of total protein, vegetable/animal protein and carbohydrate were not related to prostate cancer risk $[44,47]$. For fructose, one study showed an inverse association with prostate cancer risk [76], whereas a second study showed no association [47]. As for fat and fatty acids, of 26 associations reported in five studies, two were negative, 14 null and 10 positive (Table 4). Total fats and total fatty acids showed no association with risk of prostate cancer in serum-based studies $[24,30,39]$, but questionnaire-based studies showed a tendency for a modest positive association between total fat consumption prostate cancer risk $(R R=1.1-1.3$, Table 5$)$
$[44,47,67,79]$. For saturated fat (including animal fat) results were inconsistent, with a null [24] and positive association [39] in two serum-based studies, and one inverse [67], one null [79] and two positive $[44,66]$ associations in questionnaire-based studies. For different categorizations of unsaturated fat there were mostly null associations in serum-based studies [24,35,39], although one showed an inverse association for $n-6$ polyunsaturated fatty acids (PUFA) [39] and one a positive association for monounsaturated fat [39]. Questionnaire-based studies showed an increased risk for intake of mono-unsaturated fat $[44,67,79]$ and polyunsaturated fat [67].

Linoleic acid and $\alpha$-linolenic acid are the two fatty acids most frequently investigated for the risk of prostate cancer. For linoleic acid, serum-based studies showed either an inverse association with prostate cancer risk [32] or no effect [39]; two questionnaire-based studies also showed no effect $[44,79] . \alpha-$ Linolenic acid was positively associated with prostate cancer risk in two serum-based 
TABLE 3 Descriptive characteristics of published prospective cohort studies on dietary risk factors for prostate cancer, measured by questionnaires or interviews, and anthropometric risk factors, according to year of baseline measurement

\begin{tabular}{|c|c|c|c|c|c|c|c|c|}
\hline Country Ref & Baseline & $\begin{array}{l}\text { Cohort size } \\
\text { (age range) }\end{array}$ & $\begin{array}{l}\text { Follow-up, } \\
\text { years }\end{array}$ & $\begin{array}{l}\text { No. of cases } \\
\text { (I or D) }\end{array}$ & $\begin{array}{l}\text { Exposure } \\
\text { measure }\end{array}$ & $\begin{array}{l}\text { Investigated } \\
\text { exposure variables }\end{array}$ & $\begin{array}{l}\mathrm{N} \text { items on } \\
\text { diet (alcohol) }\end{array}$ & $\begin{array}{l}\text { Validation } \\
\text { dietary } 0\end{array}$ \\
\hline USA [53] & $1880-1916$ & 18000 (CA) & $?$ & 268 (D) & Measure & $\mathrm{AP}$ & - & - \\
\hline USA [54] & $1916-50$ & 51477 (CA) & $16-50$ & $243(I+D)$ & 0; exam & $A, A P$ & unknown & unknown \\
\hline Denmark [55] & $1954-87$ & 15214 (?) & 12.9 & $91(\mathrm{I})$ & Int & $A$ & - & - \\
\hline USA [56] & $1957-58$ & $1899(40-55)$ & 30 & $132(I+D)$ & Int; Q; DH & V & 195 & unknown \\
\hline USA [57] & 1959 & 336442 (?) & 12 & $?$ (D) & 0 & AP & - & - \\
\hline USA [58] & 1960 & $6763(>60)$ & 21 & 99 (D) & 0 & $1-3,5,6, \mathrm{AP}$ & $14 ?$ & unknown \\
\hline Japan [59] & 1965 & $122261(>40)$ & 17 & 183 (D) & Int & $1,3,5,6, A$ & $7(2)$ & unknown \\
\hline USA [60] & 1966 & $17633(>35)$ & 20 & 149 (D) & Mailed 0 & $1-6,14, A$ & 35 (2?) & unknown \\
\hline Sweden [61] & 1967 & $10942(36-75)$ & 30 & $466(I+D)$ & 0 & $1-5$ & 107 & unknown \\
\hline Sweden [62] & $1971-75$ & $135006(<30-\geq 60)$ & 20 & $2368(I+D)$ & 0; Measure & AP & & \\
\hline USA [63] & $1972-74$ & $1776(50-84)$ & 14 & $54(I)$ & Int; exam & $5,6, A P$ & 2 & unknown \\
\hline USA [64] & $1975-80$ & 20316 (>45) & 14 & $198(I)$ & Int; 0 & $1-6,14, A, A P$ & 13 food items & no \\
\hline USA $[65,66]$ & 1976 & $13855(>25)$ & $6-16$ & $180-225$ (I) & 0 & $1-6$, soy, A & 65 & unknown \\
\hline Norway [67] & 1977-83 & $25708(16-56)$ & 12.4 & $72(I)$ & FFO & $3-7, A P$ & 80 & yes \\
\hline USA [68] & $1978-85$ & 43432 (>30) & 4.6 & $238(I)$ & 0 & A & (unknown)* $^{*}$ & unknownt \\
\hline USA [69] & $1981-85$ & $11577(>60)$ & 8 & $208(I)$ & 0 & $1,2, V$ & 59 & unknown \\
\hline USA [70] & 1982 & $1050(>65)$ & 11 & $71(I)$ & Int & $A, A P$ & unknown & unknown \\
\hline USA [71] & 1982 & $450279(>30)$ & 9 & 1748 (D) & 0 & 1 & unknown & unknown \\
\hline USA [80] & 1992 & $86404(50-74)$ & 6.5 & $3739(I)$ & FFO & dairy, supplements & 68 & yes \\
\hline
\end{tabular}

Notes as for Table 2, except; CA, college attendants; Int, interview; ${ }^{0}$ Uuestions on current and past consumption, number of drinks consumed per day, and the type of alcoholic beverage; +Data compared closely to data from 7-day recall.

studies [32,39], but two questionnaire-based studies gave inconsistent results (one positive [79] and one negative [44] association). Other individual fatty acids were investigated in two serum-based $[32,39]$ and one questionnairebased [44] study; there were no consistent associations, except for oleic acid which was associated with increased prostate cancer risk in three studies $[32,39,44]$.

Table 6 summarizes results on foods and alcohol intake, measured by questionnaires or interviews. Consumption of total vegetables showed inverse associations $[60,71]$ and null associations $[42,47,69,78]$ with prostate cancer risk. Furthermore, there were inverse associations for the consumption of green salads [66] and pulses, including soy milk $[42,65,66]$. Three studies showed an inverse association between consumption of tomatoes/tomato sauce and prostate cancer risk $[66,73,78]$, but one study showed null association for tomatoes and tomato juice [42]. In contrast, positive associations with prostate cancer risk were reported for consumption of fried vegetables [24], and cooked and raw spinach [78]. Cruciferous vegetables and broccoli showed no relation with prostate cancer risk in two studies
$[42,78]$, but a positive association in a third [60]. Several other (groups of) vegetables were investigated, e.g. green and yellow vegetables $[59,69]$, raw and cooked vegetables [42], carrots [78], and alliums (onions, leeks, etc. [42]) but no association with prostate cancer risk was found.

Overall consumption of fruit showed no association with prostate cancer risk in five studies $[58,60,66,69,78]$, a positive association in three $[24,42,47]$, and a negative association in one [76]. For specific fruit items there was no association except for raisins, dates and other dried fruit, which showed a decreased prostate cancer risk [66], and citrus fruit/ oranges which showed null association in two studies $[66,78]$ but a positive association in a third [42].

Overall meat consumption was not consistently related with prostate cancer risk; three studies showed an increased risk $[58,59,66]$, one a decreased risk [67], and three no relation $[24,60,74]$. For consumption of different types of meat, including processed meats, most reported associations were positive (16) or null (19); only four studies showed a negative association (Table 6).
Consumption of fish showed no consistent relationship with prostate cancer risk: two studies $[24,66]$ showed a positive and two $[59,61]$ a negative association, and six $[45,60,64,67,72,82]$ no effect.

Consumption of milk and dairy products was either positively associated (13 reported) or unrelated (14 reported) with the risk of prostate cancer; no negative associations were reported. For milk, two positive $[58,64]$, and four null $[24,59,78,82]$ associations were reported, and for whole milk, one positive [74] and two null $[63,66]$. Several studies suggested positive associations for consumption of skimmed milk $[67,74]$; cheese [58]; butter [74]; butter, margarine and cheese combined [24]; and ice-cream $[24,74]$.

Consumption of eggs was not significantly related to prostate cancer risk in seven of 10 studies $[45,60,63,64,67,78,82]$, with a positive association observed in two of the remaining studies $[24,58]$ and an inverse association in one study [66].

Coffee consumption was investigated in four studies $[24,60,64,82]$; only one showed an 
TABLE 4 Effect of nutrient supplementation on prostate cancer risk from the results of intervention studies, and the relationship between nutrients and prostate cancer risk from prospective cohort studies using biomarkers in blood (or toenails if indicated) as a measure of exposure

\begin{tabular}{|c|c|c|c|c|c|c|}
\hline \multirow[b]{3}{*}{ Exposure } & \multicolumn{6}{|l|}{ Effect estimate } \\
\hline & \multicolumn{2}{|l|}{$<0.80$} & \multicolumn{2}{|l|}{$0.80-1.20$} & \multicolumn{2}{|l|}{$>1.20$} \\
\hline & Item & estimate $[\mathrm{ref}]^{*}$ & Item & estimate [ref] & Item & estimate [ref] \\
\hline \multicolumn{7}{|c|}{ Intervention (supplements) } \\
\hline \multirow[t]{2}{*}{ Vitamins } & $\beta$-carotene & $0.68+\neq[13]$ & $\beta$-carotene & $1.20[16], 1.0[12,14]$ & $\beta$-carotene & $1.33 \rrbracket[13]$ \\
\hline & vitamin $\mathrm{E}$ & $0.68+[16]$ & $\beta$-carotene & $1.01[17]$ & & \\
\hline Selenium & selenium & $0.37+[15]$ & + retinol & & & \\
\hline \multicolumn{7}{|l|}{ Cohort } \\
\hline \multirow[t]{10}{*}{ Vitamins } & A & $0.42+[27]$ & retinol & NS [20] & retinol & $1.56[46], 1.48$ [36], \\
\hline & retinol & $0.40[26], \mathrm{CL}[28]$ & carotenes & $0.92[36]$ & & $1.4[25], 3.0[21], \mathrm{CH}[19]$ \\
\hline & $\alpha$-carotene & $0.77[46]$ & $\beta$-carotene & $1.08[26]$ & carotenoids & $\mathrm{CH}[19]$ \\
\hline & lycopene & $0.75 / 0.57 \S[46], 0.50[26]$ & $\beta$-cryptoxanthin & $0.80[46]$ & $\beta$-carotene & $5.0+[25]$ \\
\hline & $\mathrm{D}(1,25-\mathrm{OHD})$ & $\mathrm{CL}+[29]$ & lutein & $1.10[46]$ & & \\
\hline & $E$ & 0.60 [23], CL [19] & C & $0.93[36]$ & & \\
\hline & $\alpha$-tocopherol & $0.65[48]$ & $\mathrm{D}[25-\mathrm{OHD}]$ & $0.92[34], \mathrm{CS}[29]$ & & \\
\hline & $\gamma$-tocopherol & $0.19+[48]$ & $\mathrm{D}[1,25-\mathrm{OHD}]$ & $0.88[34]$ & & \\
\hline & & & $E$ & $1.00[26]$ & & \\
\hline & & & $\alpha$-tocopherol & $1.06[46]$ & & \\
\hline \multirow[t]{2}{*}{ Selenium } & [blood] & $\mathrm{CL}[28], 0.3$ [21] & selenium [blood] & NS [40] & & \\
\hline & [toenails] & $0.58[48], 0.49+[41], 0.63+[52]$ & & & & \\
\hline \multicolumn{7}{|c|}{ Fat and fatty acids } \\
\hline & $n-6$ PUFA & 0.7 [39] & total & $0.87[24]$ & saturated & $1.6[39]$ \\
\hline & stearic & $0.35[32]$ & total fatty acids & $1.1[39], 1.05[30]^{\circ}$ & monounsaturated & $1.3[39]$ \\
\hline & arachidic & $0.7[39]$ & saturated & $1.00[24]$ & myristic & $1.8+[39]$ \\
\hline & docosanoic & $0.7[39]$ & unsaturated & $1.09[24]$ & palmitic & $2.3+[39]$ \\
\hline & tetracosanoic & $0.5+[39]$ & polyunsaturated & $1.1[39]$ & stearic & $1.3[39]$ \\
\hline & tetracosenoic & 0.7 [39] & n-6 fatty acids & NA [35] & oleic & $1.8+[39], 1.50[32]$ \\
\hline & linoleic & $0.62[32]$ & $n-3$ fatty acids & NA [35] & palmitoleic & $2.8+[39]$ \\
\hline & docosapentaenoic & 0.7 [39] & $n-3$ PUFA & $1.1[39]$ & arachidonic & $1.36[32]$ \\
\hline & & & palmitic & $0.90[32]$ & $\alpha$-linolenic & $2.0+[39], 2.14[32]$ \\
\hline & & & linoleic & 0.9 [39] & & \\
\hline & & & dihomo- $\boldsymbol{\gamma}$-linoleic & $1.1[39]$ & & \\
\hline & & & arachidonic & $0.8[39]$ & & \\
\hline & & & eicosenoic & $1.2[39]$ & & \\
\hline & & & eicosadienoic & $1.0[39]$ & & \\
\hline & & & eicosapentaenoic & $1.2[39], 0.87[32]$ & & \\
\hline & & & docosahexaenoic & $1.0[39]$ & & \\
\hline
\end{tabular}

*Effect estimates for active vs placebo group (intervention studies) or for highest vs lowest exposure category (cohort studies); +denotes statistically significant finding. In patient quartile with $\neq$ lowest or $\$$ highest baseline plasma $\beta$-carotene levels. CL, cases with lower blood levels than controls; $C S$, cases with similar blood level to controls; $\mathrm{CH}$, cases with higher blood level than controls; NA, not associated; $\$ A$ djusted for assignment to $\beta$-carotene treatment or placebo; ${ }^{\circ}$ triglycerides, mmol/L; PUFA, polyunsaturated fatty acids; NS, not significantly associated.

association with prostate cancer risk, which was positive [82]. Tea was only reported in one study, and showed no association for black tea and a borderline significant increase in risk for green tea [24].

Ten studies were identified in which the relation between consumption of alcoholic beverages and prostate cancer risk was investigated. Overall consumption of alcohol was not associated with risk of prostate cancer in six studies $[24,43,64,68,70,82]$, but was associated with an increased risk in two $[55,59]$ and a decreased risk in one study [66]. Whereas specific types of alcoholic beverages were not associated with risk of prostate cancer in most studies [59,60,82], one showed a negative association for alcohol from beer, but significant positive associations for alcohol from wine, white wine and fortified wines; the only study investigating red wine showed no association [43].

Table 7 presents results from prospective cohort studies on anthropometric variables, e.g. height, weight and body mass index (BMI) in relation to the risk of prostate cancer. In all, 19 positive, 24 null and three negative associations were reported between anthropometric variables and prostate cancer 
TABLE 5 Relation between nutrients and prostate cancer risk from prospective cohort studies using dietary questionnaires or interviews to measure exposure

\begin{tabular}{|c|c|c|c|c|c|c|}
\hline \multirow[b]{3}{*}{ Exposure } & \multicolumn{6}{|l|}{ Effect estimate } \\
\hline & \multicolumn{2}{|l|}{$<0.80$} & \multicolumn{2}{|l|}{$0.80-1.20$} & \multicolumn{2}{|l|}{$>1.20$} \\
\hline & Item & estimate $\left[\right.$ ref] ${ }^{*}$ & Item & estimate [ref] & Item & estimate [ref] \\
\hline \multirow[t]{14}{*}{ Vitamins } & A (supp) & $0.65[51]$ & A & $1.13[78], 1.1[60]$ & retinol & $1.32+[78]$ \\
\hline & lycopene & $0.79+[78]$ & A (supp) & 1.13 [69] & $\beta$-cryptoxanthin & $1.45+[51]$ \\
\hline & & & retinol & $0.93[51], 1.2[60]$ & c & $1.22[51], 1.27[56]$ \\
\hline & E (supp) & $0.71[51]$ & $\alpha$-carotene & $0.85[51], 1.09$ [78] & & \\
\hline & & & $\beta$-carotene & $\begin{array}{l}1.07[51], 1.05[78], 1.09[69] \\
0.9[60]\end{array}$ & & \\
\hline & & & Iycopene & $0.94[73], 1.03$ [56] & & \\
\hline & & & $\beta$-cryptoxanthin & $0.94[78], 1.03[56]$ & & \\
\hline & & & lutein & $1.10[78]$ & & \\
\hline & & & lutein/zeaxanthin & $0.91[51]$ & & \\
\hline & & & C & 1.09 [78], 0.96 [69] & & \\
\hline & & & C (supp) & $1.00[69], 0.83[51]$ & & \\
\hline & & & $\mathrm{D}$ & 0.8 [47], NAt+ [80] & & \\
\hline & & & $E$ & 0.95 [51], NS [40], 0.94 [78] & & \\
\hline & & & E (supp) & 1.05 [75], NS [40], 1.00 [69] & & \\
\hline \multirow[t]{3}{*}{ Calcium } & & & Calcium & $1.09[45], 1.1[47], 1.2+[80]$ & Calcium & $1.71[76]$ \\
\hline & & & Calcium (supp) & $1.1[80]$ & Calcium (diet) & $1.6+[80]$ \\
\hline & & & & & Calcium (dairy prod) & $1.34+[50]$ \\
\hline \multirow[t]{3}{*}{ Protein } & & & Protein & $1.0[47], 1.09[45]$ & & \\
\hline & & & Protein, vegetable & $0.92[45]$ & & \\
\hline & & & Protein, animal & $1.16[45]$ & & \\
\hline \multirow[t]{13}{*}{ Fat } & saturated & 0.7 [67] & total fat & $1.11[44], 1.1[47]$ & total fat & $1.32[79], 1.3[67]$ \\
\hline & linolenic acid & $0.76[44]$ & total fatty acids & $1.15[44]$ & saturated FA & $1.23[44]$ \\
\hline & & & saturated fat & $0.84[79]$ & MU fat & $1.4[67], 1.86$ [79] \\
\hline & & & linoleic & $0.88[79]$ & MUFA & $1.22[44]$ \\
\hline & & & poly UFA & $0.80[44]$ & polyunsaturated fat & $1.4[67]$ \\
\hline & & & trans UFA & $1.01[44]$ & animal fat & $1.35[66]$ \\
\hline & & & cis UFA & $0.89[44]$ & oleic & $1.30[44]$ \\
\hline & & & palmitic & $1.16[44]$ & $\alpha$-linolenic & $1.25[79]$ \\
\hline & & & stearic & $1.17[44]$ & & \\
\hline & & & linoleic & $0.80[44]$ & & \\
\hline & & & arachidonic & $1.20[44]$ & & \\
\hline & & & eicosapentaenoic & $1.01[44]$ & & \\
\hline & & & docosahexaenoic & $1.05[44]$ & & \\
\hline \multirow[t]{2}{*}{ Carbohydrate } & fructose & $0.77[76]$ & carbohydrate & $1.1[47]$ & & \\
\hline & & & fructose & $1.0[47]$ & & \\
\hline
\end{tabular}

Notes as for Table 4. UFA, unsaturated fatty acids; MU, monounsturated; FA, fatty acids; supp, supplement; t+Advanced prostate cancer (Stages C and D).

risk. In four studies a there was a statistically significant positive association between height and prostate cancer risk $[37,62,64,77]$, whereas in four others there was no association $[30,53,54,68,70]$. Weight showed either positive $[31,57,58,62,70]$ or null associations $[53,64,68]$. There were positive associations between $\mathrm{BMI}$ at baseline and prostate cancer risk in four studies $[22,30,67,70]$ whereas six showed no association $[49,62,63,66,68,77]$; two showed a positive association for $\mathrm{BMI}$ when young adults (20-25 years) $[49,70]$, but a third showed no effect [77]. An increase in BMI when aged $25-65$ years (reflecting weight gain over this period) was negatively associated with prostate cancer risk in two studies $[49,70]$, but not associated in a third [54]. Some other measures of body fat distribution, e.g. lean body mass, thickness of skinfolds or waist/hip circumference, were investigated with either null $[22,49,53,62,77]$ or positive [54] associations.
Only a few of all prospective studies investigated latent and nonlatent or advanced prostate tumours separately $[24,29,32,39,43,44,49,64,70,72,74,77-79,83]$. For some risk factors, e.g. vitamin D [29], total fat, mono-unsaturated fat and $\alpha$-linolenic acid [79], meat [74], red meat [45], reported associations were stronger for advanced or aggressive prostate tumours, whereas for fish, a negative association was reported for metastatic prostate cancer. For other risk factors like carotenoids [78], fat and fatty 
TABLE 6 Foods and alcohol in relation to prostate cancer risk from prospective cohort studies using questionnaires or interviews to measure exposure

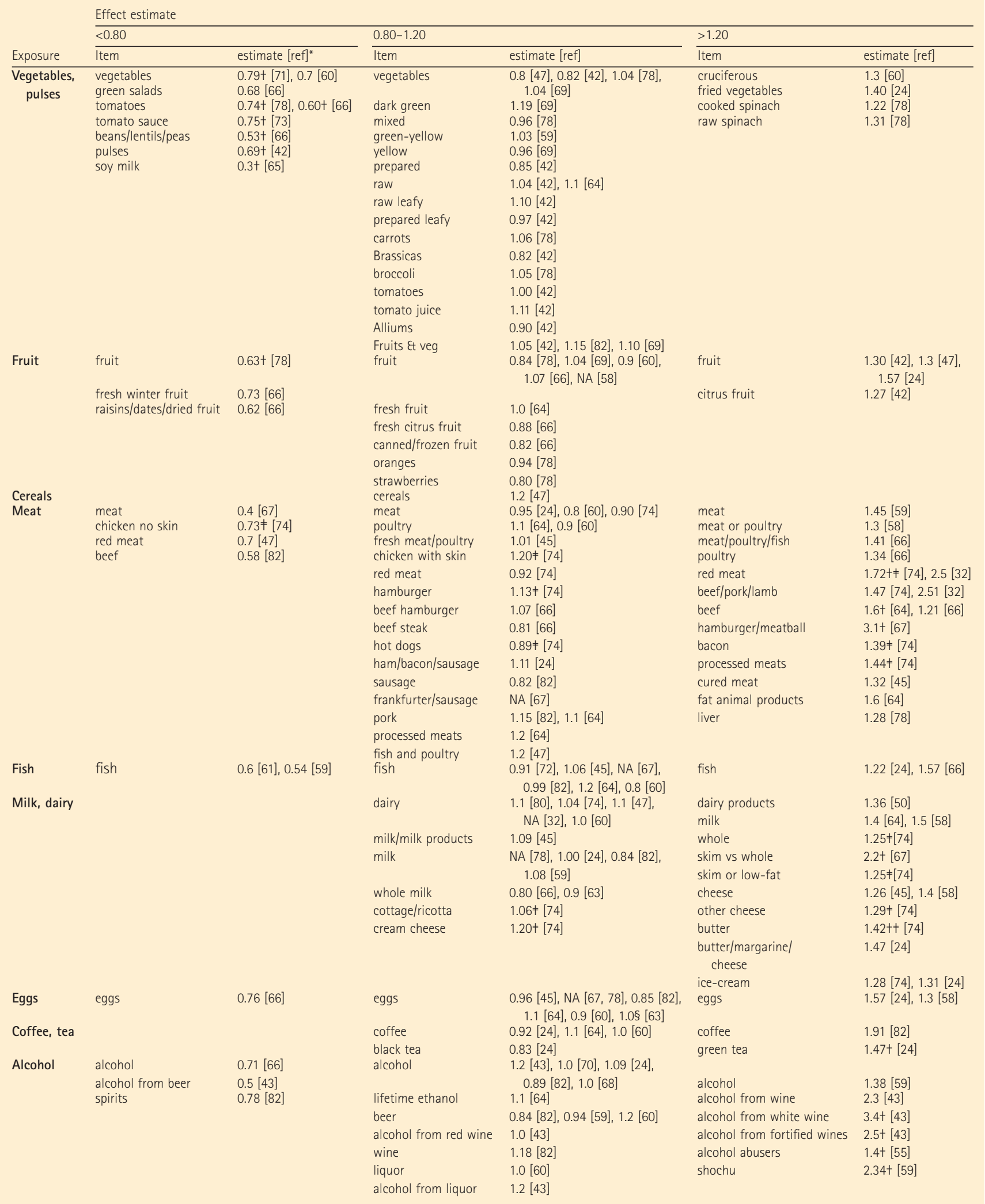


TABLE 7 Relation between anthropometric measures and prostate cancer risk from prospective cohort studies

\begin{tabular}{|c|c|c|c|c|c|c|}
\hline \multirow[b]{3}{*}{ Exposure } & \multicolumn{6}{|l|}{ Effect estimate } \\
\hline & \multicolumn{2}{|l|}{$<0.80$} & \multicolumn{2}{|l|}{$0.80-1.20$} & \multicolumn{2}{|l|}{$>1.20$} \\
\hline & Item & estimate [ref] & Item & estimate [ref] & Item & estimate [ref] \\
\hline \multirow[t]{15}{*}{$\begin{array}{c}\text { Height, weight, } \\
\text { BMI, LBM }\end{array}$} & BMI & $0.7[64]$ & height & $\begin{array}{r}0.97[49]_{1}, 1.14+[62]_{1} 1.1[70], \\
0.99 \neq[30], N A[53,54,68]\end{array}$ & height & $\begin{array}{l}1.37+[77], 1.27+[37], \\
1.8+[64]\end{array}$ \\
\hline & $\begin{array}{l}\text { change in } \mathrm{BMI} \\
\text { from age } 25 \\
\text { to }>65\end{array}$ & $\begin{array}{l}0.61[49] \\
0.7[70]\end{array}$ & & & leg length & $1.24[22]$ \\
\hline & & & sitting height & $0.94[22]$ & & \\
\hline & & & weight & $1.16+[62], 0.9[64]$, NA $[53,68]$ & weight & $\begin{array}{l}1.6[70], 1.52+[31]_{1} \\
1.29[57], 2.4+[58]\end{array}$ \\
\hline & & & $\mathrm{BMI}$ & $\begin{array}{r}0.85[49], 0.90[77], 1.13[62] \\
\text { NA [68], } 1.17[66] 1.19[63]\end{array}$ & $\mathrm{BMI}$ & $\begin{array}{l}1.5[70]_{,} 1.33[22]_{1} \\
2.2+[67], 1.25+[30]\end{array}$ \\
\hline & & & Ponderal index & NA [53] & $\mathrm{BMI}$ at age 20 & $1.34[49]$ \\
\hline & & & BMI at age 21 & $0.87[77]$ & $\mathrm{BMI}$ at age 25 & $1.4[70]$ \\
\hline & & & change in $\mathrm{BMI}$ during & NA [54] & $\mathrm{BMI}$ at age 50 & $1.3[70]$ \\
\hline & & & life & & $\mathrm{BMI}$ change 50 to $>65$ & $1.3[70]$ \\
\hline & & & & & girth left upper arm & $1.49[22]$ \\
\hline & & & LBM & $0.96[49], 1.17[62]$ & scapular skinfold & $1.27[22]$ \\
\hline & & & triceps skinfold & $0.94[22]$ & thickness & \\
\hline & & & thickness & & biacromial diam & $1.22[22]$ \\
\hline & & & waist circumference & $0.99[77]$ & & \\
\hline & & & hip circumference & $0.85[77]$ & & \\
\hline
\end{tabular}

fPer $10 \mathrm{~cm}$; Per $2.92 \mathrm{~kg} / \mathrm{m} 2$. Other notes as for Table 4; BMI, Body Mass Index; LBM, Lean Body Mass.

acids [44], vegetables and fruit [42], fried vegetables and fruit [24], beef and high-fat animal products [64], milk and eggs [24,45], alcohol [43] and BMI [49,70], reported associations were either similar in subgroups based on tumour characterization, or were inconsistent among studies.

\section{DISCUSSION}

We present an overview of the epidemiological evidence on the potential role of dietary factors in the aetiology of prostate cancer. This review was restricted to prospective studies for two reasons; first, results of case-control studies were inconclusive $[11,84]$ and second, such studies are prone to both selection and information bias. In all, 41 studies (37 cohort, four intervention) published until September 2003 were included. Given the high incidence of prostate cancer and the largely unknown causes of the disease, it seems probable that any findings on possible risk factors would be published; therefore, the possibility of publication bias is less likely. Furthermore, to minimize this type of bias, qualitative findings mentioned in publications (i.e. with no effect estimates) were also included in the review.

Before discussing the specific findings two drawbacks must be noted; first, despite considerable research efforts over the past decades, the available evidence for many individual foods and nutrients, e.g. specific fatty acids, types of vegetables and fruits, alcoholic beverages, processed meats, specific dairy products, etc., remains limited, which hampers firm conclusions on such foods or nutrients at present. Second, for several foods or food groups for which more information is available, this review shows no or inconsistent associations with prostate cancer risk. In the absence of a true association, random variation may lead to some studies with false-positive $(R R>1)$ and some studies with false-negative $(R R<1)$ associations, which are evenly distributed around $\mathrm{RR} \approx 1$. However, inconsistent results of different studies may also be caused by heterogeneity in study size, outcome measures, duration of follow-up, extensiveness of dietary evaluation, and identification of potential confounders. Some of these factors will be briefly discussed below.

Many studies were small; 38\% included $<100$ cases; $43 \%$ used prostate cancer mortality, or a combination of incidence and mortality, as an endpoint. In general, in aetiological studies incidence is a more appropriate endpoint than mortality, as mortality is determined by many factors, including tumour stage and oncological treatment. In only five studies was the validation of dietary questionnaires reported; the relatively crude dietary exposure information collected by questionnaires/ interviews in the remaining studies may have resulted in random misclassification of dietary exposure to different foods and nutrients. Inadequacies in food composition tables are another potential source of error in questionnaire-based studies. Although several studies used blood samples as a biomarker of long-term dietary intake, this method is also limited by the short half-life of several nutrients (e.g. vitamin C) and between-subject variability in the absorption, metabolism and excretion of nutrients. Generally, random misclassification of exposure could have 
resulted in underestimation of true positive/ negative associations between diet and prostate cancer risk.

Another potential cause of inconsistent results of different studies is bias by confounding. As only a minority of the prospective studies had an extensive dietary assessment, controlling for potential confounding by other dietary components was not possible in most cases. However, for $\approx 15$ publications reporting both crude and multivariate adjusted Risk ratios, we compared these values. In virtually all cases, RRs changed only marginally after multivariate adjustment, indicating that confounding did not play a major role.

Inconsistent results between studies may well be caused by differences in study population between countries, e.g. the USA, Finland and the Netherlands. For instance, in the USA, many foods are fortified with vitamins, leading to a different food matrix when compared with studies in Finland or the Netherlands. Also, that three of four intervention studies were in high-risk groups or smokers may impede extrapolation to the general population. Finally, differences in aetiology between latent and clinically apparent or advanced/aggressive prostate tumours may have influenced the reported associations between dietary factors and prostate cancer risk, an issue which was not addressed in most studies.

Nevertheless, considering all these limitations, the main findings of this review can be summarized as follows.

- Although overall consumption of meat and fish appears to be unrelated to prostate cancer risk, most studies show either null or positive associations for different types of meat, and this also applies to milk and dairy products. For eggs, most studies suggest no relationship with prostate cancer risk.

- A potential protective effect of vegetables is supported by only two of six studies, whereas four show no relation. Although data on pulses are limited, all three available cohort studies consistently show a protective effect. Furthermore, three out of four cohort studies support a protective role for tomatoes/tomato sauce. Cruciferous vegetables and alliums (onion, leek, etc.) appear to be unrelated to prostate cancer risk. - Overall fruit consumption is not associated with prostate cancer risk in five studies, but with an increased risk in three and a reduced risk in one. Of individual fruits, only dried fruit is associated with reduced risk in one study [66].

- As for vitamin A and carotenoids, four intervention studies consistently failed to show any protective effect of $\beta$-carotene on prostate cancer; two studies show no effect, one an insignificant increase, and one a reduction in subjects with low serum levels of $\beta$-carotene at baseline, but an increase in subjects with high baseline serum $\beta$-carotene levels. Cohort studies also show no consistent associations of vitamin $A$ and carotenoids with prostate cancer risk, except for lycopene: two plasma-based and one questionnairebased cohort studies of lycopene suggest a negative association, but this relation was not detected in two other questionnaire-based studies.

- For vitamin $E_{1}$ one intervention study [16] indicates a reduction of prostate cancer risk by vitamin E supplementation. This is supported by three serum-based cohort studies, but not by a fourth, nor by questionnaire-based studies of vitamin $E$ intake from food or supplements. Data on vitamins $C$ and $D$ show no consistent effect. - Selenium shows protective effects in one intervention study [15]; this is consistent with $a \approx 50 \%$ risk reduction by high vs low selenium in toenails or blood in five of six cohort studies. For calcium, although two cohort studies show no association, two other studies show an increased prostate cancer risk at high intake (2000 mg/day); there were independent associations for calcium from foods and supplements.

- For fat, most associations reported in five cohort studies are null or positive. For total fat (or total fatty acids), three serum-based studies show no effect; in contrast, four questionnaire-based studies show RRs of 1.1-1.3, suggesting a slightly increased risk of prostate cancer for subjects with high fat consumption. For individual fatty acids, data are highly inconsistent, except an elevated risk ( $R R=1.2-2.8)$ for mono-unsaturated fatty acids (including oleic and palmitoleic acid) in two studies.

- Three of four studies on coffee consumption show no association with prostate cancer risk, but one an increased risk. The only study on tea consumption does not suggest a relationship with prostate cancer risk.

- Studies on alcohol consumption show, again, no consistent relation between overall alcohol consumption and risk of prostate cancer. For individual alcoholic beverages, most studies show a either null association or an increased risk.

- Findings for anthropometric variables suggest either a null or a positive association of height, BMI and other anthropometric variables with prostate cancer risk. In conclusion, this review of prospective studies on diet and prostate cancer risk indicates a protective effect for selenium; other potentially protective foods/nutrients may be vitamin $E_{1}$ pulses and tomatoes/ lycopene. Overall consumption of meat and consumption of eggs, vegetables, fruit, coffee, tea, carotenoids, and the vitamins $A, C$ and $D$ show no consistent relations with prostate cancer risk. For specific types of both meat and alcoholic beverages, as well as for fat and dairy products, mostly null or positive associations are reported; this also applies to anthropometric measures. Furthermore, two cohort studies suggest that very high calcium intake ( $>2000 \mathrm{mg} /$ day) may be associated with increased prostate cancer risk.

Future research on dietary risk factors for prostate cancer should be based on an extensive exposure measurement including information on both dietary and non-dietary risk factors. With time the increasing numbers of cases will increase the reliability of findings, and allow subgroup analyses.

\section{REFERENCES}

1 Boyle $\mathbf{P}$, Maisonneuve $\mathrm{P}$, Napalkov $\mathrm{P}$. Geographical and temporal patterns of incidence and mortality from prostate cancer. Urology 1995; 46 (Suppl. A): 47-55

2 Parkin DM, Muir CS, Whelan SL, Gao Y-T, Ferlay J, Powell J. Cancer incidence in five continents. Lyon: IARC Scientific Publications, 1992

3 Coffey DS. Prostate cancer. An overview of an increasing dilemma. Cancer 1993; 71 (Suppl.): 880-6

4 Pienta KJ, Esper PS. Risk factors for prostate cancer. Ann Intern Med 1993; 118: 793-803

5 Zaridze DG, Boyle P. Cancer of the prostate: epidemiology and aetiology. Br J Urol 1987; 59: 493-502

6 Boyle P, Severi G, Giles GG. The epidemiology of prostate cancer. Urol Clin North Am 2003; 30: 209-17

7 Shirai T, Asamoto M, Takahashi S, Imaida K. Diet and prostate cancer. Toxicology 2002; 181-182: 89-94 
Nelson MA, Reid M, Duffield-Lillico AJ, Marshall JR. Prostate cancer and selenium. Urol Clin North Am 2002; 29: 67-70

9 Jankevicius F, Miller SM, Ackermann R. Nutrition and risk of prostate cancer. Urol Int 2002; 68: 69-80

10 Yip I, Heber D, Aronson W. Nutrition and prostate cancer. Urol Clin North Am 1999; 26: 403-11

11 Giles G, Ireland P. Diet, nutrition and prostate cancer. Int J Cancer 1997; 10 (Suppl): 13-7

12 Cook NR, Le IM, Manson JE, Buring JE, Hennekens $\mathrm{CH}$. Effects of beta-carotene supplementation on cancer incidence by baseline characteristics in the Physicians' Health Study (United States). Cancer Causes Control 2000; 11: 617-26

13 Cook NR, Stampfer MJ, Ma J et al. Betacarotene supplementation for patients with low baseline levels and decreased risks of total and prostate carcinoma. Cancer 1999; 86: 1783-92

14 Hennekens $\mathrm{CH}$, Buring JE, Manson JE et al. Lack of effect of long-term supplementation with beta carotene on the incidence of malignant neoplasms and cardiovascular disease. N Engl J Med 1996; 334: 1145-9

15 Clark LC, Dalkin B, Krongrad A et al. Decreased incidence of prostate cancer with selenium supplementation: results of a double-blind cancer prevention trial. $\mathrm{Br}$ J Urol 1998; 81: 730-4

16 Heinonen OP, Albanes D, Virtamo J et al. Prostate cancer and supplementation with alpha-tocopherol and betacarotene: incidence and mortality in a controlled trial. J Natl Cancer Inst 1998; 90: 440-6

17 Omenn GS, Goodman GE, Thornquist MD et al. Risk factors for lung cancer and for intervention effects in CARET, the Beta-Carotene and Retinol Efficacy Trial. J Natl Cancer Inst 1996; 88: 1550-9

18 Kark JD, Smith AH, Switzer BR, Hames CG. Serum vitamin A (retinol) and cancer incidence in Evans County, Georgia. J Natl Cancer Inst 1981; 66: 7-16

19 Willett WC, Polk BF, Underwood BA et al. Relation of serum vitamins $A$ and $E$ and carotenoids to the risk of cancer. N Engl J Med 1984; 310: 430-4

20 Peleg I, Heyden S, Knowles M, Hames CG. Serum retinol and risk of subsequent cancer: extension of the Evans County, Georgia, study. J Natl Cancer Inst 1984; 73: $1455-8$
Coates RJ, Weiss NS, Daling JR, Morris JS, Labbe RF. Serum levels of selenium and retinol and the subsequent risk of cancer. Am J Epidemiol 1988; 128: 51523

22 Severson RK, Grove JS, Nomura AM, Stemmermann GN. Body mass and prostatic cancer: a prospective study. Br Med J 1988; 297: 713-5

23 Knekt P, Aromaa A, Maatela J et al. Serum vitamin $E$ and risk of cancer among Finnish men during a 10-year follow-up. Am J Epidemiol 1988; 127: 28-41

24 Severson RK, Nomura AM, Grove JS, Stemmermann GN. A prospective study of demographics, diet, and prostate cancer among men of Japanese ancestry in Hawaii. Cancer Res 1989; 49: 1857-60

5 Knekt P, Aromaa A, Maatela J et al. Serum vitamin $A$ and subsequent risk of cancer: cancer incidence follow-up of the Finnish Mobile Clinic Health Examination Survey. Am J Epidemiol 1990; 132: 85770

26 Hsing AW, Comstock GW, Abbey H, Polk BF. Serologic precursors of cancer. Retinol, carotenoids, and tocopherol and risk of prostate cancer. J Nat/ Cancer Inst 1990; 82: 941-6

Reichman ME, Hayes RB, Ziegler RG et al. Serum vitamin $A$ and subsequent development of prostate cancer in the first National Health and Nutrition Examination Survey Epidemiologic Follow-up Study. Cancer Res 1990; 50: 2311-5

28 Criqui MH, Bangdiwala S, Goodman DS et al. Selenium, retinol, retinol-binding protein, and uric acid. Associations with cancer mortality in a population-based prospective case-control study. Ann Epidemiol 1991; 1: 385-93

29 Corder EH, Guess HA, Hulka BS et al. Vitamin $D$ and prostate cancer: a prediagnostic study with stored sera. Cancer Epidemiol Biomarkers Prev 1993; 2: 467-72

30 Thune I, Lund E. Physical activity and the risk of prostate and testicular cancer: a cohort study of 53,000 Norwegian men. Cancer Causes Control 1994; 5: 549-56

31 Chyou PH, Nomura AM, Stemmermann GN. A prospective study of weight, body mass index and other anthropometric measurements in relation to site-specific cancers. Int J Cancer 1994; 57: 313-7

32 Gann PH, Hennekens CH, Sacks FM, Grodstein F, Giovannucci EL, Stampfer MJ. Prospective study of plasma fatty acids and risk of prostate cancer. J Natl Cancer Inst 1994; 86: 281-6

33 Corder EH, Friedman GD, Vogelman JH, Orentreich N. Seasonal variation in vitamin D, vitamin D-binding protein, and dehydroepiandrosterone: risk of prostate cancer in black and white men. Cancer Epidemiol Biomarkers Prev 1995; 4: 655-9

34 Gann PH, Ma J, Hennekens $\mathrm{CH}$, Hollis BW, Haddad JG, Stampfer MJ.

Circulating vitamin D metabolites in relation to subsequent development of prostate cancer. Cancer Epidemiol Biomarkers Prev 1996; 5: 121-6

5 Alberg AJ, Kafonek S, Huang HY, Hoffman SC, Comstock GW, HelzIsouer $\mathrm{KJ}$. Fatty acid levels and the subsequent development of prostate cancer. Proc Am Assoc Cancer Res 1996; 37: 281

36 Eichholzer M, Stahelin HB, Gey KF, Ludin E, Bernasconi F. Prediction of male cancer mortality by plasma levels of interacting vitamins: 17-year follow-up of the prospective Basel study. Int J Cancer 1996; 66: 145-50

37 Hebert PR, Ajani U, Cook NR, Lee IM, Chan KS, Hennekens $\mathrm{CH}$. Adult height and incidence of cancer in male physicians (United States). Cancer Causes Control 1997; 8: 591-7

38 Nomura AM, Stemmermann GN, Lee J, Craft NE. Serum micronutrients and prostate cancer in Japanese Americans in Hawaii. Cancer Epidemiol Biomarkers Prev 1997; 6: 487-91

39 Harvei S, Bjerve KS, Tretli S, Jellum E, Robsahm TE, Vatten L. Prediagnostic level of fatty acids in serum phospholipids: omega-3 and omega- 6 fatty acids and the risk of prostate cancer. Int J Cancer 1997; 71: 545-51

40 Hartman TJ, Albanes D, Peitinen P et al. The association between baseline vitamin $E$, selenium, and prostate cancer in the Alpha-Tocopherol, Beta-Carotene Prevention Study. Cancer Epidemiol Biomarker Prev 1998; 7: 335-40

41 Yoshizawa K, Willett WC, Morris SJ et al. Study of prediagnostic selenium level in toenails and the risk of advanced prostate cancer. J Natl Cancer Inst 1998; 90: 1219-24

42 Schuurman AG, Goldbohm RA, Dorant E, Van den Brandt PA. Vegetable and fruit consumption and prostate cancer risk: a cohort study in the Netherlands. Cancer Epidemiol Biom Prev 1998; 7: 67380

43 Schuurman AG, Goldbohm RA, van den 
Brandt PA. A prospective cohort study on consumption of alcoholic beverages in relation to prostate cancer incidence (The Netherlands). Cancer Causes Control 1999; 10: 597-605

44. Schuurman AG, van den Brandt PA, Dorant E, Brants HA, Goldbohm RA. Association of energy and fat intake with prostate carcinoma risk: results from The Netherlands Cohort Study. Cancer 1999; 86: 1019-27

45 Schuurman AG, van den Brandt PA, Dorant E, Goldbohm RA. Animal products, calcium and protein and prostate cancer risk in The Netherlands Cohort Study. Br J Cancer 1999; 80: 110713

46 Gann PH, Ma J, Giovannucci E et al. Lower prostate cancer risk in men with elevated plasma lycopene levels: results of a prospective analysis. Cancer Res 1999; 59: $1225-30$

47 Chan JM, Pietinen P, Virtanen M et al. Diet and prostate cancer risk in a cohort of smokers, with a specific focus on calcium and phosphorus (Finland). Cancer Causes Control 2000; 11: 859-67

48 Helzlsouer KJ, Huang HY, Alberg AJ et al. Association between alphatocopherol, gamma-tocopherol, selenium, and subsequent prostate cancer. J Natl Cancer Inst 2000; 92: 2018-23

49 Schuurman AG, Goldbohm RA, Dorant $\mathrm{E}$, van den Brandt PA. Anthropometry in relation to prostate cancer risk in the Netherlands Cohort Study. Am J Epidemiol 2000; 151: 541-9

50 Chan JM, Stampfer MJ, Ma J, Gann PH, Gaziano JM, Giovannucci EL. Dairy products, calcium, and prostate cancer risk in the Physicians' Health Study. Am J Clin Nutr 2001; 74: 549-54

51 Schuurman AG, Goldbohm RA, Brants $H A$, van den Brandt PA. A prospective cohort study on intake of retinol, vitamins $C$ and $E_{1}$ and carotenoids and prostate cancer risk (Netherlands). Cancer Causes Control 2002; 13: 573-82

52 van den Brandt PA, Zeegers MP, Bode P, Goldbohm RA. Toenail selenium levels and the subsequent risk of prostate cancer: a prospective cohort study. Cancer Epidemiol Biomarkers Prev 2003; 12: 866-71

53 Greenwald P, Damon A, Kirmss V, Polan AK. Physical and demographic features of men before developing cancer of the prostate. J Natl Cancer Inst 1974; 53: $341-6$
54 Whittemore AS, Paffenbarger RS Jr, Anderson K, Lee JE. Early precursors of site-specific cancers in college men and women. J Natl Cancer Inst 1985; 74: $43-$ 51

55 Tonnesen $\mathrm{H}$, Moller $\mathrm{H}$, Andersen JR,

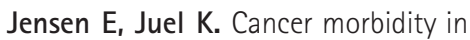
alcohol abusers. Br J Cancer 1994; 69: 327-32

56 Daviglus ML, Dyer AR, Persky V et al. Dietary beta-carotene, vitamin $C_{\text {, }}$ and risk of prostate cancer: results from the Western Electric Study. Epidemiology 1996; 7: 472-7

57 Garfinkel L. Overweight and mortality. Cancer 1986; 58 (Suppl.): 1826-9

58 Snowdon DA, Phillips RL, Choi W. Diet, obesity, and risk of fatal prostate cancer. Am J Epidemiol 1984; 120: 244-50

59 Hirayama T. Life-style and mortality. A large-scale census-based cohort study in Japan. Basel: Karger, 1990

60 Hsing AW, McLaughlin JK, Schuman LM et al. Diet, tobacco use, and fatal prostate cancer: results from the Lutheran Brotherhood Cohort Study. Cancer Res 1990; 50: 6836-40

61 Terry $\mathrm{P}$, Lichtenstein P, Feychting M, Ahlbom A, Wolk A. Fatty fish consumption and risk of prostate cancer. Lancet 2001; 357: 1764-6

62 Andersson SO, Wolk A, Bergstrom R et al. Body size and prostate cancer: a 20year follow-up study among 135006 Swedish construction workers. J Natl Cancer Inst 1997; 89: 385-9

63 Thompson MM, Garland C, BarrettConnor E, Khaw KT, Friedlander NJ, Wingard DL. Heart disease risk factors, diabetes, and prostatic cancer in an adult community. Am J Epidemiol 1989; 129: $511-7$

64 Le Marchand L, Kolonel LN, Wilkens LR, Myers BC, Hirohata T. Animal fat consumption and prostate cancer: a prospective study in Hawaii. Epidemiology 1994; 5: 276-82

65 Jacobsen BK, Knutsen SF, Fraser GE. Does high soy milk intake reduce prostate cancer incidence? The Adventist Healt Study (United States). Cancer Causes Control 1998; 9: 553-7

66 Mills PK, Beeson WL, Phillips RL, Fraser GE. Cohort study of diet, lifestyle, and prostate cancer in Adventist men. Cancer 1989; 64: 598-604

67 Veierod MB, Laake P, Thelle DS. Dietary fat intake and risk of prostate cancer: a prospective study of 25,708
Norwegian men. Int J Cancer 1997; 73: 634-8

68 Hiatt RA, Armstrong MA, Klatsky AL, Sidney S. Alcohol consumption, smoking, and other risk factors and prostate cancer in a large health plan cohort in California (United States). Cancer Causes Control 1994; 5: 66-72

69 Shibata A, Paganini Hill A, Ross RK, Henderson BE. Intake of vegetables, fruits, beta-carotene, vitamin $\mathrm{C}$ and vitamin supplements and cancer incidence among the elderly: a prospective study. Br J Cancer 1992; 66: 673-9

70 Cerhan JR, Torner JC, Lynch CF et al. Association of smoking, body mass, and physical activity with risk of prostate cancer in the lowa 65+ Rural Health Study (United States). Cancer Causes Control 1997; 8: 229-38

71 Rodriguez C, Tatham LM, Thun MJ, Calle EE, Heath CW Jr. Smoking and fatal prostate cancer in a large cohort of adult men. Am J Epidemiol 1997; 145: 466-75

72 Augustsson K, Michaud DS, Rimm EB et al. A prospective study of intake of fish and marine fatty acids and prostate cancer. Cancer Epidemiol Biomarkers Prev 2003; 12: 64-7

73 Giovannucci E, Rimm EB, Liu Y, Stampfer MJ, Willett WC. A prospective study of tomato products, lycopene, and prostate cancer risk. J Natl Cancer Inst 2002; 94: 391-8

74 Michaud DS, Augustsson K, Rimm EB, Stampfer MJ, Willet WC, Giovannucci E. A prospective study on intake of animal products and risk of prostate cancer. Cancer Causes Control 2001; 12: 557-67

75 Chan JM, Stampfer MJ, Ma J, Rimm EB, Willett WC, Giovannucci EL. Supplemental vitamin $\mathrm{E}$ intake and prostate cancer risk in a large cohort of men in the United States. Cancer Epidemiol Biomarkers Prev 1999; 8: 893-9

76 Giovannucci E, Rimm EB, Wolk A et al. Calcium and fructose intake in relation to risk of prostate cancer. Cancer Res 1998; 58: $442-7$

77 Giovannucci E, Rimm EB, Stampfer MJ, Colditz GA, Willett WC. Height, body weight, and risk of prostate cancer. Cancer Epidemiol Biomarkers Prev 1997; 6: 557-63

78 Giovannucci E, Ascherio A, Rimm EB, Stampfer MJ, Colditz GA, Willett WC. Intake of carotenoids and retinol in 


\section{P.C. DAGNELIE ET AL.}

relation to risk of prostate cancer. J Natl Cancer Inst 1995; 87: 1767-76

79 Giovannucci E, Rimm EB, Colditz GA et al. A prospective study of dietary fat and risk of prostate cancer. J Nat/ Cancer Inst 1993; 85: 1571-9

80 Rodriguez C, McCullough ML, Mondul AM et al. Calcium, dairy products, and risk of prostate cancer in a prospective cohort of United States men. Cancer Epidemiol Biomarkers Prev 2003; 12: 597-603

81 Clark LC, Combs GF Jr, Turnbull BW et al. Effects of selenium supplementation for cancer prevention in patients with carcinoma of the skin. A randomized controlled trial. Nutritional Prevention
Cancer Study Group. JAMA 1996; 276: 1957-63

82 Gronberg H, Damber L, Damber JE. Total food consumption and body mass index in relation to prostate cancer risk: a case-control study in Sweden with prospectively collected exposure data. J Urol 1996; 155: 969-74

83 Hsing AW, Comstock GW. Serological precursors of cancer: serum hormones and risk of subsequent prostate cancer. Cancer Epidemiol Biomarkers Prev 1993; 2: 27-32

84 Kolonel LN. Nutrition and prostate cancer. Cancer Causes Control 1996; 7: 83-94

85 Terry PD, Rohan TE, Wolk A. Intakes of fish and marine fatty acids and the risks of cancers of the breast and prostate and of other hormone-related cancers: a review of the epidemiologic evidence. Am J Clin Nutr 2003; 77: 532-43

Correspondence: P.C. Dagnelie, PhD, Department of Epidemiology, Maastricht University, PO Box 616, 6200 MD Maastricht, the Netherlands.

e-mail: Dagnelie@epid.unimaas.nl

Abbreviations: $\mathrm{RR}$, relative risk; $\mathrm{ATBC}$, AlphaTocopherol Beta-Carotene Cancer Prevention Study; PHS, Physicians' Health Study; PUFA, polyunsaturated fatty acids; BMI, body mass index. 